\title{
Proximity Theorems of Discrete Convex Functions
}

\author{
東京大学・情報理工学研究科 室田 一雄 (Kazuo Murota) \\ Graduate School of Information Science and Technology, University of Tokyo \\ 京都大学 - 数理解析研究所 田村 明久 (Akihisa Tamura) \\ Research Institute for Mathematical Sciences, Kyoto University
}

\begin{abstract}
A proximity theorem is a statement that, given an optimization problem and its relaxation, an optimal solution to the original problem exists in a certain neighborhood of a solution to the relaxation. Proximity theorems have been used successfully, for example, in designing efficient algorithms for discrete resource allocation problems. After reviewing the recent results for L-convex and M-convex functions, this paper establishes proximity theorems for larger classes of discrete convex functions, $\mathrm{L}_{2}$-convex functions and $\mathrm{M}_{2}$-convex functions, that are relevant to the polymatroid intersection problem and the submodular flow problem.
\end{abstract}

\section{Introduction}

In the area of discrete optimization, nonlinear optimization problems have been investigated as well as linear optimization problems. Submodular (set) functions and separable convex functions are well-known examples of tractable nonlinear functions, in that the submodular function minimization problem can be solved in polynomial time (see [13, 14, 24]), and separable convex functions have been treated successfully in many different discrete optimization problems (see [11]).

Recently, certain classes of "discrete convex functions" were proposed: $\left\{\mathrm{L}, \mathrm{M}, \mathrm{L}_{2}, \mathrm{M}_{2}\right\}$ convex functions of Murota [18, 19]. L-convex functions contain the class of submodular set functions. M-convex functions possess structures of matroids and polymatroids. Separable discrete convex functions can be characterized as functions with both L-convexity and $\mathrm{M}$-convexity (in their variants). $\mathrm{L}_{2}$-convex functions and $\mathrm{M}_{2}$-convex functions constitute larger classes of discrete convex functions that are relevant to the polymatroid intersection problem, where an $\mathrm{L}_{2}$-convex function is, by definition, the infimal convolution of two L-convex functions and an $\mathrm{M}_{2}$-convex function is the sum of two $\mathrm{M}$-convex functions. The $\mathrm{M}_{2}$-convex function minimization problem is equivalent to the $\mathrm{M}$-convex submodular flow problem [20] which is an extension of the submodular flow problem [3].

Those classes $C$ of discrete convex functions $f$ possess the following features in com- 
Discreteness: $f$ is defined on an integral lattice $\mathbf{Z}^{n}$, i.e., $f: \mathbf{Z}^{n} \rightarrow \mathbf{R} \cup\{+\infty\}$, where $\mathbf{Z}$ and $\mathbf{R}$ denote the sets of integers and reals, respectively.

Convex Extendibility: There exists a continuous convex function $\bar{f}$ such that $\bar{f}(x)=$ $f(x)$ for all $x \in \mathbf{Z}^{n}$.

Optimality Criterion: There exists a neighborhood $N_{C}\left(x^{*}\right) \subset \mathbf{Z}^{n}$ with center $x^{*}$ such that

$$
f\left(x^{*}\right) \leq f(x)\left(\forall x \in \mathbf{Z}^{n}\right) \Leftrightarrow f\left(x^{*}\right) \leq f(x)\left(\forall x \in N_{C}\left(x^{*}\right)\right)
$$

Optimality criterion says that global minimality is implied by local minimality defined in terms of the neighborhood $N_{C}\left(x^{*}\right)$. This is a significant feature inherited from continuous convex functions.

Moreover, L-/M-convex functions have a "proximity property" described as

Proximity Property: Given a positive integer $\alpha$ and a point $x^{\alpha} \in \mathbf{Z}^{n}$, there exists a function $d_{C}(n, \alpha)$ such that

$$
f\left(x^{\alpha}\right) \leq f(x)\left(\forall x \in N_{C}^{\alpha}\left(x^{\alpha}\right)\right) \Rightarrow \exists x^{*} \in \arg \min f:\left\|x^{*}-x^{\alpha}\right\|_{\infty} \leq d_{C}(n, \alpha),
$$

where $N_{C}^{\alpha}\left(x^{\alpha}\right)=\left\{x^{\alpha}+\alpha\left(x-x^{\alpha}\right) \mid x \in N_{C}\left(x^{\alpha}\right)\right\}$ and $\arg \min f$ denotes the set of all minimizers of $f$, i.e.,

$$
\arg \min f=\left\{x \in \mathbf{Z}^{n} \mid f(x) \leq f(y)\left(\forall y \in \mathbf{Z}^{n}\right)\right\} .
$$

The proximity property says that a locally minimal solution $x^{\alpha}$ of a "scaled" function

$$
f^{\alpha}(x)=f\left(x^{\alpha}+\alpha x\right) \quad\left(x \in \mathbf{Z}^{V}\right)
$$

is close to a minimizer $x^{*}$ of $f$ in terms of $d_{C}(n, \alpha)$. For L-/M-convex functions, $d_{C}(n, \alpha)=$ $(n-1)(\alpha-1)$ is a valid choice ([15] and [16], respectively). The proximity property can be exploited in developing an efficient scaling algorithm for minimizing $f$. In fact, the L-convex function minimization problem can be solved in polynomial-time by combining submodular set function minimization algorithms and the proximity property [12] (see also [21]). For the M-convex function minimization, polynomial-time scaling algorithms based on the proximity property and its generalization are known [25, 26]. Proximity theorems for separable discrete convex functions are found in $[8,9,17]$ in developing efficient algorithms for resource allocation problems. Different types of theorems on proximity have also been investigated: proximity between integral and real optimal solutions in $[1,2,7,9,10]$ and proximity for a number of resource allocation problems with min-max type objective functions in [5].

This paper addresses proximity properties of $\mathrm{L}_{2}-/ \mathrm{M}_{2}$-convex functions. Our main results say: 
- for an essentially bounded $\mathrm{L}_{2}$-convex function $f$ and a positive integer $\alpha$, if $x^{\alpha} \in$ $\operatorname{dom} f$ satisfies

$$
f\left(x^{\alpha}\right) \leq f\left(x^{\alpha}+\alpha \chi_{S}\right)
$$

for all $S \subseteq V$, then there exists $x^{*} \in \arg \min f$ such that

$$
\left\|x^{*}-x^{\alpha}\right\|_{\infty} \leq 2(n-1)(\alpha-1)
$$

- for an $\mathrm{M}_{2}$-convex function $f$ represented as the sum of two M-convex functions $f_{1}$ and $f_{2}$, and a positive integer $\alpha$, if $x^{\alpha} \in \operatorname{dom} f$ satisfies

$$
\sum_{i=1}^{k}\left(f_{1}\left(x^{\alpha}-\alpha \chi_{u_{i}}+\alpha \chi_{w_{i}}\right)-f_{1}\left(x^{\alpha}\right)\right)+\sum_{i=1}^{k}\left(f_{2}\left(x^{\alpha}-\alpha \chi_{u_{i+1}}+\alpha \chi_{w_{i}}\right)-f_{2}\left(x^{\alpha}\right)\right) \geq 0
$$

for any ordered sets $U=\left\{u_{1}, \ldots, u_{k}\right\}, W=\left\{w_{1}, \ldots, w_{k}\right\} \subset V$ with $U \cap W=\emptyset$ where $u_{k+1}=u_{1}$, then there exists $x^{*} \in \arg \min f$ such that

$$
\left\|x^{*}-x^{\alpha}\right\|_{\infty} \leq \frac{n^{2}}{2}(\alpha-1) .
$$

Section 2 states definitions, optimality criteria and proximity properties for several classes of discrete convex functions.

\section{Definitions, Optimality Criteria and Proximity Theorems}

In this section, we introduce four classes of discrete convex functions, namely, $\{\mathrm{L}, \mathrm{M}$, $\mathrm{L}_{2}, \mathrm{M}_{2}$ \}-convex functions with respect to definitions, optimality criteria and proximity theorems. While other variants of these classes, e.g., $L^{\natural}-/ L_{2}^{\natural}$-convex functions due to [6] and $\mathrm{M}^{\mathrm{h}}-/ \mathrm{M}_{2}^{\natural}$-convex functions due to [22], are known, we concentrate on the above four classes because the results can be easily extended to the variants.

Subsections 2.3 and 2.4 present new results, an optimality criterion (Theorem 2.8) and a proximity property (Theorem 2.9 ) for $\mathrm{L}_{2}$-convex functions, and proximity properties (Theorems 2.12 and 2.13) for $\mathrm{M}_{2}$-convex functions. Subsection 2.2 also gives a new proximity property (Theorem 2.6) for M-convex functions in terms of $\ell_{1}$-norm. Subsections 2.1 and 2.2 explain known results, optimality criteria and proximity theorems for L-convexity and M-convexity, respectively. Subsection 2.4 introduce optimality criteria for $\mathrm{M}_{2}$-convexity, which are direct consequences of results for the M-convex submodular flow problem.

We first introduce notations. Let $V$ be a nonempty finite set and put $n=|V|$. We denote by $\mathbf{Z}^{V}$ the set of all integral vectors $x=(x(v): v \in V)$ indexed by $V$, and by $\mathbf{Z}_{++}$the set of all positive integers. Given a function $f: \mathbf{Z}^{V} \rightarrow \mathbf{R} \cup\{ \pm \infty\}$, the effective domain of $f$ is defined by

$$
\operatorname{dom} f=\left\{x \in \mathbf{Z}^{V} \mid f(x) \neq \pm \infty\right\}
$$


For each $S \subseteq V$, we denote by $\chi_{S}$ the characteristic vector of $S$ defined by

$$
\chi_{S}(v)=\left\{\begin{array}{ll}
1 & (v \in S) \\
0 & (v \notin S)
\end{array} \quad(v \in V)\right.
$$

and write simply $\chi_{u}$ instead of $\chi_{\{u\}}$ for each $u \in V$. We also denote by 0 and 1 the vectors of all zeros and ones, respectively. For two vectors $x, y \in \mathbf{Z}^{V}$ with $x \leq y,[x, y]_{\mathbf{z}}$ denotes the set $\left\{z \in \mathbf{Z}^{V} \mid x \leq z \leq y\right\}$.

\subsection{L-convex Functions}

For any $x, y \in \mathbf{Z}^{V}$, the vectors $x \wedge y$ and $x \vee y$ in $\mathbf{Z}^{V}$ are such that

$$
(x \wedge y)(v)=\min \{x(v), y(v)\}, \quad(x \vee y)(v)=\max \{x(v), y(v)\} \quad(v \in V) .
$$

A function $f: \mathbf{Z}^{V} \rightarrow \mathbf{R} \cup\{+\infty\}$ is said to be $L$-convex if $\operatorname{dom} f \neq \emptyset$ and it satisfies the following two conditions:

(SBF) $f$ is submodular, i.e.,

$$
f(x)+f(y) \geq f(x \wedge y)+f(x \vee y) \quad\left(\forall x, y \in \mathbf{Z}^{V}\right),
$$

(TRF) $\exists r \in \mathbf{R}$ such that $f(x+\mathbf{1})=f(x)+r \quad\left(\forall x \in \mathbf{Z}^{V}\right)$.

Global optimality of an L-convex function is characterized by local optimality.

Theorem 2.1 (L-optimality criterion, [21])

For an L-convex function $f: \mathbf{Z}^{V} \rightarrow \mathbf{R} \cup\{+\infty\}$ and $x^{*} \in \operatorname{dom} f$, we have

$$
f\left(x^{*}\right) \leq f(x) \quad\left(\forall x \in \mathbf{Z}^{V}\right) \Longleftrightarrow\left\{\begin{array}{l}
f\left(x^{*}\right) \leq f\left(x^{*}+\chi_{S}\right) \quad(\forall S \subseteq V), \\
f\left(x^{*}+\mathbf{1}\right)=f\left(x^{*}\right) .
\end{array}\right.
$$

The above local optimality criterion can be checked in polynomial time because the first condition can be verified by using submodular function minimization algorithms and the second condition is easy.

We next introduce a proximity theorem of L-convex functions.

Theorem 2.2 (L-proximity theorem, [15])

Let $f: \mathbf{Z}^{V} \rightarrow \mathbf{R} \cup\{+\infty\}$ be an L-convex function with $f(x+\mathbf{1})=f(x)\left(\forall x \in \mathbf{Z}^{V}\right)$ and let $\alpha \in \mathbf{Z}_{++}$. If $x^{\alpha} \in \operatorname{dom} f$ satisfies

$$
f\left(x^{\alpha}\right) \leq f\left(x^{\alpha}+\alpha \chi_{S}\right) \quad(\forall S \subseteq V),
$$

then $\arg \min f \neq \emptyset$ and there exists $x^{*} \in \arg \min f$ with

$$
x^{\alpha} \leq x^{*} \leq x^{\alpha}+(n-1)(\alpha-1) \mathbf{1} .
$$

Remark 2.3 Theorems 2.1 and 2.2 are extended to a more general class of "quasi" Lconvex functions [23]. 


\subsection{M-convex Functions}

We define the positive support and negative support of a vector $x=(x(v): v \in V) \in \mathbf{Z}^{V}$ by

$$
\operatorname{supp}^{+}(x)=\{v \in V \mid x(v)>0\} \quad \text { and } \quad \operatorname{supp}^{-}(x)=\{v \in V \mid x(v)<0\} .
$$

A function $f: \mathbf{Z}^{V} \rightarrow \mathbf{R} \cup\{+\infty\}$ is called M-convex if $\operatorname{dom} f \neq \emptyset$ and it satisfies

(M-EXC) for $x, y \in \operatorname{dom} f$ and $u \in \operatorname{supp}^{+}(x-y)$, there exists $v \in \operatorname{supp}^{-}(x-y)$ such that

$$
f(x)+f(y) \geq f\left(x-\chi_{u}+\chi_{v}\right)+f\left(y+\chi_{u}-\chi_{v}\right) .
$$

We note that (M-EXC) is also represented as: for $x, y \in \operatorname{dom} f$,

$$
f(x)+f(y) \geq \max _{u \in \operatorname{supp}^{+}(x-y)} \min _{v \in \operatorname{supp}^{-}(x-y)}\left[f\left(x-\chi_{u}+\chi_{v}\right)+f\left(y+\chi_{u}-\chi_{v}\right)\right],
$$

where the maximum and the minimum over an empty set are $-\infty$ and $+\infty$, respectively. From (M-EXC), the effective domain $\operatorname{dom} f$ lies on a hyperplane $\left\{x \in \mathbf{R}^{V} \mid x(V)=\right.$ constant\}, where $x(V)=\sum_{v \in V} x(v)$. It is also known that $\operatorname{dom} f$ is the set of integer points of the base polyhedron of an integral submodular system (see [4] for submodular systems).

The minimizers of an M-convex function have a nice characterization which can be checked efficiently.

Theorem 2.4 (M-optimality criterion, $[18,19]$ )

For an $M$-convex function $f: \mathbf{Z}^{V} \rightarrow \mathbf{R} \cup\{+\infty\}$ and $x^{*} \in \operatorname{dom} f$, we have

$$
f\left(x^{*}\right) \leq f(x)\left(\forall x \in \mathbf{Z}^{V}\right) \quad \Longleftrightarrow \quad f\left(x^{*}\right) \leq f\left(x^{*}-\chi_{u}+\chi_{v}\right) \quad(\forall u, v \in V) .
$$

We next introduce a proximity theorem of M-convex functions.

Theorem 2.5 (M-proximity theorem, [16])

Let $f: \mathbf{Z}^{V} \rightarrow \mathbf{R} \cup\{+\infty\}$ be an $M$-convex function and let $\alpha \in \mathbf{Z}_{++}$. If $x^{\alpha} \in \operatorname{dom} f$ satisfies

$$
f\left(x^{\alpha}\right) \leq f\left(x^{\alpha}-\alpha \chi_{u}+\alpha \chi_{v}\right) \quad(\forall u, v \subseteq V),
$$

then $\arg \min f \neq \emptyset$ and there exists $x^{*} \in \arg \min f$ with

$$
\left|x^{\alpha}(v)-x^{*}(v)\right| \leq(n-1)(\alpha-1) \quad(\forall v \in V) .
$$

By slightly modifying the proof of [16], we also obtain the following proximity theorem in terms of $\ell_{1}$-norm. 
Theorem 2.6 Let $f: \mathbf{Z}^{V} \rightarrow \mathbf{R} \cup\{+\infty\}$ be an $M$-convex function and let $\alpha \in \mathbf{Z}_{++}$. If $x^{\alpha} \in \operatorname{dom} f$ satisfies

$$
f\left(x^{\alpha}\right) \leq f\left(x^{\alpha}-\alpha \chi_{u}+\alpha \chi_{v}\right) \quad(\forall u, v \subseteq V)
$$

then $\arg \min f \neq \emptyset$ and there exists $x^{*} \in \arg \min f$ with

$$
\left\|x^{*}-x^{\alpha}\right\|_{1} \leq \frac{n^{2}}{2}(\alpha-1)
$$

Remark 2.7 Theorems 2.4 and 2.5 are extended to a more general class of "quasi" Mconvex functions [23].

\section{$2.3 \quad \mathrm{~L}_{2}$-convex Functions}

For any functions $f_{1}, f_{2}: \mathbf{Z}^{V} \rightarrow \mathbf{R} \cup\{+\infty\}$, the infimal convolution of $f_{1}$ and $f_{2}$, denoted by $f_{1} \square f_{2}: \mathbf{Z}^{V} \rightarrow \mathbf{R} \cup\{ \pm \infty\}$, is defined by

$$
\left(f_{1} \square f_{2}\right)(x)=\inf \left\{f_{1}\left(x_{1}\right)+f_{2}\left(x_{2}\right) \mid x_{1}+x_{2}=x, x_{1}, x_{2} \in \mathbf{Z}^{V}\right\} \quad\left(x \in \mathbf{Z}^{V}\right) .
$$

It is easy to show that if $f_{1} \square f_{2}>-\infty$ then the effective domain of $f_{1} \square f_{2}$ coincides with the Minkowski sum of the effective domains of $f_{1}$ and $f_{2}$, that is,

$$
\operatorname{dom}\left(f_{1} \square f_{2}\right)=\left(\operatorname{dom} f_{1}\right)+\left(\operatorname{dom} f_{2}\right) \equiv\left\{x_{1}+x_{2} \mid x_{1} \in \operatorname{dom} f_{1}, x_{2} \in \operatorname{dom} f_{2}\right\}
$$

It is known that the infimal convolution of two M-convex functions is also M-convex, but the infimal convolution of two L-convex functions may not be L-convex [18]. A function $f: \mathbf{Z}^{V} \rightarrow \mathbf{R} \cup\{+\infty\}$ is said to be $L_{2}$-convex if $\operatorname{dom} f \neq \emptyset$ and $f=f_{1} \square f_{2}$ for some L-convex functions $f_{1}, f_{2}: \mathbf{Z}^{V} \rightarrow \mathbf{R} \cup\{+\infty\}$. We say that an L-/ $\mathrm{L}_{2}$-convex function $f$ is essentially bounded if $\operatorname{dom} f \cap\left\{x \in \mathbf{Z}^{V} \mid x(v)=0\right\}$ is bounded for some $v \in V$. If an $\mathrm{L}_{2}$-convex function $f=f_{1} \square f_{2}$ is essentially bounded, then $f_{1}$ and $f_{2}$ are also essentially bounded, because $\operatorname{dom} f=\left(\operatorname{dom} f_{1}\right)+\left(\operatorname{dom} f_{2}\right)$ holds for $\mathrm{L}_{2}$-convex function $f$.

The following optimality criterion and the proximity theorem for $\mathrm{L}_{2}$-convex functions are new results. We emphasize that the optimality criterion is the same as that for Lconvex functions stated in Theorem 2.1 and that the proximity theorem is almost the same as that stated in Theorem 2.2 .

\section{Theorem 2.8 ( $\mathrm{L}_{2}$-optimality criterion)}

For an $L_{2}$-convex function $f: \mathbf{Z}^{V} \rightarrow \mathbf{R} \cup\{+\infty\}$ and $x^{*} \in \operatorname{dom} f$, we have

$$
f\left(x^{*}\right) \leq f(x) \quad\left(\forall x \in \mathbf{Z}^{V}\right) \Longleftrightarrow\left\{\begin{array}{l}
f\left(x^{*}\right) \leq f\left(x^{*}+\chi_{S}\right) \quad(\forall S \subseteq V), \\
f\left(x^{*}+1\right)=f\left(x^{*}\right)
\end{array}\right.
$$


Theorem 2.9 ( $\mathrm{L}_{2}$-proximity theorem)

Let $f: \mathbf{Z}^{V} \rightarrow \mathbf{R} \cup\{+\infty\}$ be an essentially bounded $L_{2}$-convex function with $f(x+\mathbf{1})=$ $f(x)\left(\forall x \in \mathbf{Z}^{V}\right)$ and let $\alpha \in \mathbf{Z}_{++}$. If $x^{\alpha} \in \operatorname{dom} f$ satisfies

$$
f\left(x^{\alpha}\right) \leq f\left(x^{\alpha}+\alpha \chi_{S}\right) \quad(\forall S \subseteq V),
$$

then $\arg \min f \neq \emptyset$ and there exists $x^{*} \in \arg \min f$ with

$$
x^{\alpha} \leq x^{*} \leq x^{\alpha}+2(n-1)(\alpha-1) \mathbf{1}
$$

\section{$2.4 \quad M_{2}$-convex Functions}

It is known that the sum of two M-convex functions is not necessarily M-convex. A function $f: \mathbf{Z}^{V} \rightarrow \mathbf{R} \cup\{+\infty\}$ is said to be $M_{2}$-convex if $\operatorname{dom} f \neq \emptyset$ and $f=f_{1}+\dot{f}_{2}$ for some M-convex functions $f_{1}, f_{2}: \mathbf{Z}^{V} \rightarrow \mathbf{R} \cup\{+\infty\}$. It is easy to show that $\operatorname{dom} f=$ $\left(\operatorname{dom} f_{1}\right) \cap\left(\operatorname{dom} f_{2}\right)$. Obviously, if $\operatorname{dom} f_{1}=\operatorname{dom} f_{2}$ and $f_{2}$ is identically zero, then $f=f_{1}$ is $\mathrm{M}$-convex, and hence, the class of $\mathrm{M}_{2}$-convex functions includes that of $\mathrm{M}$-convex functions. The $\mathrm{M}_{2}$-convex function minimization problem contains the polymatroid intersection problem as a special case. Thus, optimality criteria for $\mathrm{M}_{2}$-convexity below are extensions of known results for the matroid intersection problem and the polymatroid intersection problem.

For a vector $p \in \mathbf{R}^{V}$, let us define functions $\langle p, x\rangle$ and $f[p](x)$ by

$$
\langle p, x\rangle=\sum_{v \in V} p(v) x(v) \quad \text { and } \quad f[p](x)=f(x)+\langle p, x\rangle \quad\left(x \in \mathbf{Z}^{V}\right) .
$$

If $f$ is M-convex, then $f[p]$ is also M-convex.

Several results on optimality of $\mathrm{M}_{2}$-convexity are known.

Theorem 2.10 (M-convex intersection theorem, [18])

For $M$-convex functions $f_{1}, f_{2}: \mathbf{Z}^{V} \rightarrow \mathbf{R} \cup\{+\infty\}$ and a point $x^{*} \in \operatorname{dom} f_{1} \cap \operatorname{dom} f_{2}$, we have

$$
f_{1}\left(x^{*}\right)+f_{2}\left(x^{*}\right) \leq f_{1}(x)+f_{2}(x) \quad\left(\forall x \in \mathbf{Z}^{V}\right)
$$

if and only if there exists $p^{*} \in \mathbf{R}^{V}$ such that

$$
\begin{aligned}
& f_{1}\left[-p^{*}\right]\left(x^{*}\right) \leq f_{1}\left[-p^{*}\right](x) \quad\left(\forall x \in \mathbf{Z}^{V}\right), \\
& f_{2}\left[+p^{*}\right]\left(x^{*}\right) \leq f_{1}\left[+p^{*}\right](x) \quad\left(\forall x \in \mathbf{Z}^{V}\right),
\end{aligned}
$$

and furthermore, we have

$$
\arg \min \left(f_{1}+f_{2}\right)=\arg \min \left(f_{1}\left[-p^{*}\right]\right) \cap \arg \min \left(f_{2}\left[+p^{*}\right]\right)
$$

for such $p^{*}$. 
Optimality criteria of $\mathrm{M}_{2}$-convex functions can be transformed from those of the Mconvex submodular flow problem in [19], because the $\mathrm{M}_{2}$-convex function minimization and the M-convex submodular flow problem are equivalent to each other. The following theorem is a direct consequence of the results in [19].

Theorem $2.11\left(\mathrm{M}_{2}\right.$-optimality criteria, see [19])

For $M$-convex functions $f_{1}, f_{2}: \mathbf{Z}^{V} \rightarrow \mathbf{R} \cup\{+\infty\}$ and a point $x^{*} \in \operatorname{dom} f_{1} \cap \operatorname{dom} f_{2}$, three conditions below are equivalent:

(a) $x^{*} \in \arg \min \left(f_{1}+f_{2}\right)$.

(b) For any ordered sets $U=\left\{u_{1}, \ldots, u_{k}\right\}, W=\left\{w_{1}, \ldots, w_{k}\right\} \subset V$ with $U \cap W=\emptyset$,

$$
\sum_{i=1}^{k}\left(f_{1}\left(x^{*}-\chi_{u_{i}}+\chi_{w_{i}}\right)-f_{1}\left(x^{*}\right)\right)+\sum_{i=1}^{k}\left(f_{2}\left(x^{*}-\chi_{u_{i+1}}+\chi_{w_{i}}\right)-f_{2}\left(x^{*}\right)\right) \geq 0,
$$

where $u_{k+1}=u_{1}$.

(c) $\left(f_{1}+f_{2}\right)\left(x^{*}\right) \leq\left(f_{1}+f_{2}\right)\left(x^{*}-\chi_{U}+\chi_{W}\right) \quad(\forall U, W \subset V,|U|=|W|)$.

The optimality for $\mathrm{M}_{2}$-convexity can be checked in polynomial time by transforming (b) of Theorem 2.11 to a network problem (see Remark 2.14), although checking condition

(c) of Theorem 2.11 seems to be a hard problem. In view of polynomial time verifiability, we relax (b) of Theorem 2.11 to formulate a proximity theorem of $\mathrm{M}_{2}$-convex functions. This is the main result of this paper.

\section{Theorem 2.12 ( $\mathrm{M}_{2}$-proximity theorem)}

Let $f_{1}, f_{2}: \mathbf{Z}^{V} \rightarrow \mathbf{R} \cup\{+\infty\}$ be $M$-convex functions and let $\alpha \in \mathbf{Z}_{++}$. If $x^{\alpha} \in$ $\operatorname{dom} f_{1} \cap \operatorname{dom} f_{2}$ satisfies

$$
\sum_{i=1}^{k}\left(f_{1}\left(x^{\alpha}-\alpha \chi_{u_{i}}+\alpha \chi_{w_{i}}\right)-f_{1}\left(x^{\alpha}\right)\right)+\sum_{i=1}^{k}\left(f_{2}\left(x^{\alpha}-\alpha \chi_{u_{i+1}}+\alpha \chi_{w_{i}}\right)-f_{2}\left(x^{\alpha}\right)\right) \geq 0
$$

for any ordered sets $U=\left\{u_{1}, \ldots, u_{k}\right\}, W=\left\{w_{1}, \ldots, w_{k}\right\} \subset V$ with $U \cap W=\emptyset$ where $u_{k+1}=$ $u_{1}$, then $\arg \min \left(f_{1}+f_{2}\right) \neq \emptyset$ and there exists $x^{*} \in \arg \min \left(f_{1}+f_{2}\right)$ with

$$
\left\|x^{*}-x^{\alpha}\right\|_{\infty} \leq \frac{n^{2}}{2}(\alpha-1) .
$$

The proof of Theorem 2.12 relies heavily on the following result.

Theorem 2.13 Let $f_{1}, f_{2}: \mathbf{Z}^{V} \rightarrow \mathbf{R} \cup\{+\infty\}$ be M-convex functions with $\arg \min \left(f_{1}+\right.$ $\left.f_{2}\right) \neq \emptyset$. For a given point $x \in \mathbf{Z}^{V}$ with $x(V)=y(V)$ for any $y \in \operatorname{dom} f_{1} \cap \operatorname{dom} f_{2}$, and for $d \in \mathbf{Z}$, if there exist $x^{1} \in \arg \min f_{1}$ and $x^{2} \in \arg \min f_{2}$ such that

$$
\left\|x^{1}-x\right\|_{1} \leq d, \quad\left\|x^{2}-x\right\|_{1} \leq d,
$$

then there exists $x^{*} \in \arg \min \left(f_{1}+f_{2}\right)$ with

$$
\left\|x^{*}-x\right\|_{\infty} \leq d
$$


Remark 2.14 Condition (b) of Theorem 2.11 can be checked in polynomial time. Given two M-convex functions $f_{1}, f_{2}: \mathbf{Z}^{V} \rightarrow \mathbf{R} \cup\{+\infty\}$, a point $x \in \operatorname{dom} f_{1} \cap \operatorname{dom} f_{2}$ and a positive integer $\alpha \in \mathbf{Z}_{++}$, we construct a directed graph $G_{x}^{\alpha}=\left(V_{1} \cup V_{2}, A\right)$ and an arc length $\ell_{x}^{\alpha} \in \mathbf{R}^{A}$ as follows. Let $V_{1}$ and $V_{2}$ be copies of $V$, i.e.,

$$
V_{1}=\left\{v_{1} \mid v \in V\right\}, \quad V_{2}=\left\{v_{2} \mid v \in V\right\}
$$

where $v_{1}$ and $v_{2}$ are the copies of $v \in V$. Arc set $A$ consists of three disjoint parts:

$$
\begin{aligned}
& A_{b}=\left\{\left(v_{1}, v_{2}\right) \mid v \in V\right\} \cup\left\{\left(v_{2}, v_{1}\right) \mid v \in V\right\}, \\
& A_{1}=\left\{\left(u_{1}, v_{1}\right) \mid u, v \in V, u \neq v, x-\alpha \chi_{u}+\alpha \chi_{v} \in \operatorname{dom} f_{1}\right\}, \\
& A_{2}=\left\{\left(v_{2}, u_{2}\right) \mid u, v \in V, u \neq v, x-\alpha \chi_{u}+\alpha \chi_{v} \in \operatorname{dom} f_{2}\right\} .
\end{aligned}
$$

We define $\ell_{x}^{\alpha} \in \mathbf{R}^{A}$ by

$$
\ell_{x}^{\alpha}(a)=\left\{\begin{array}{cl}
0 & \left(a \in A_{b}\right) \\
f_{1}\left(x-\alpha \chi_{u}+\alpha \chi_{v}\right)-f_{1}(x) & \left(a=\left(u_{1}, v_{1}\right) \in A_{1}\right) \\
f_{2}\left(x-\alpha \chi_{u}+\alpha \chi_{v}\right)-f_{2}(x) & \left(a=\left(v_{2}, u_{2}\right) \in A_{2}\right) .
\end{array}\right.
$$

Lemma 2.15 below guarantees that (b) of Theorem 2.11 can be checked in polynomial time by applying shortest path algorithms.

Lemma 2.15 For two M-convex functions $f_{1}, f_{2}: \mathbf{Z}^{V} \rightarrow \mathbf{R} \cup\{+\infty\}$, a point $x \in$ $\operatorname{dom} f_{1} \cap \operatorname{dom} f_{2}$ and $\alpha \in \mathbf{Z}_{++}$, two conditions below are equivalent:

(a) There exists no negative cycle in $G_{x}^{\alpha}$ with length $\ell_{x}^{\alpha}$.

(b) For any ordered sets $U=\left\{u_{1}, \ldots, u_{k}\right\}, W=\left\{w_{1}, \ldots, w_{k}\right\} \subset V$ with $U \cap W=\emptyset$,

$$
\sum_{i=1}^{k}\left(f_{1}\left(x-\alpha \chi_{u_{i}}+\alpha \chi_{w_{i}}\right)-f_{1}(x)\right)+\sum_{i=1}^{k}\left(f_{2}\left(x-\alpha \chi_{u_{i+1}}+\alpha \chi_{w_{i}}\right)-f_{2}(x)\right) \geq 0,
$$

where $u_{k+1}=u_{1}$.

\section{References}

[1] R. Baldick, Refined proximity and sensitivity results in linearly constrained convex separable integer programming, Linear Algebra Appl. 226/228 (1995), 389-407.

[2] W. Cook, A. M. H. Gerards, A. Schrijver and E. Tardos, Sensitivity theorems in integer linear programming, Math. Program. 34 (1986), 251-264.

[3] J. Edmonds and R. Giles, A min-max relation for submodular functions on graphs, Ann. Discrete Math. 1 (1977), 185-204. 
[4] S. Fujishige, "Submodular Functions and Optimization," Annals of Discrete Mathematics 47, North-Holland, Amsterdam, 1991.

[5] S. Fujishige, N. Katoh and T. Ichimori, The fair resource allocation problem with submodular constraints, Math. Oper. Res. 13 (1988), 164-173.

[6] S. Fujishige and K. Murota, Notes on L-/M-convex functions and the separation theorems, Math. Program. 88 (2000), 129-146.

[7] F. Granot and J. Skorin-Kapov, Some proximity and sensitivity results in quadratic integer programming, Math. Program. 47 (1990), 259-268.

[8] D. S. Hochbaum, Lower and upper bounds for the allocation problem and other nonlinear optimization problems, Math. Oper. Res. 19 (1994), 390-409.

[9] D. S. Hochbaum and J. G. Shanthikumar, Convex separable optimization is not much harder than linear optimization, J. Assoc. Comput. Mach. 37 (1990), 843-862.

[10] T. Ibaraki and N. Katoh, "Resource Allocation Problems: Algorithmic Approaches," The MIT Press, Cambridge, 1988.

[11] T. Ibaraki and N. Katoh, Resource Allocation Problems, in "Handbook of Combinatorial Optimization (Vol. 2)," (D.-Z. Du and P. M. Pardalos, Eds.), pp. 159-260, Kluwer Academic Publishers, Boston, 1998.

[12] S. Iwata, Oral presentation at Workshop on Matroids, Matching, and Extensions, University of Waterloo, December, 1999.

[13] S. Iwata, A fully combinatorial algorithm for submodular function minimization, $J$. Combin. Theory Ser. B 84 (2002), 203-212.

[14] S. Iwata, L. Fleischer and S. Fujishige, A combinatorial strongly polynomial algorithm for minimizing submodular functions, J. Assoc. Comput. Mach. 48 (2001), 761-777.

[15] S. Iwata and M. Shigeno, Conjugate scaling algorithm for Fenchel-type duality in discrete convex optimization, SIAM J. Optim., to appear.

[16] S. Moriguchi, K. Murota and A. Shioura, Scaling algorithms for M-convex function minimization, IEICE Trans. Fundamentals E85-A (2002), 922-929.

[17] S. Moriguchi and A. Shioura, On Hochbaum's scaling algorithm for the general resource allocation problem, Research Report B-377, Tokyo Institute of Technology, 
[18] K. Murota, Convexity and Steinitz's exchange property, Adv. Math. 124 (1996), 272-311.

[19] K. Murota, Discrete convex analysis, Math. Program. 83 (1998), 313-371.

[20] K. Murota, Submodular flow problem with a nonseparable cost function, Combinatorica 19 (1999), 87-109.

[21] K. Murota, "Discrete Convex Analysis - An Introduction (in Japanese)," Kyoritsu Publ. Co., Tokyo, 2001.

[22] K. Murota and A. Shioura, M-convex function on generalized polymatroid, Math. Oper. Res. 24 (1999), 95-105.

[23] K. Murota and A. Shioura, Quasi M-convex and L-convex functions: Quasi-convexity in discrete optimization, Discrete Appl. Math. to appear.

[24] A. Schrijver, A combinatorial algorithm minimizing submodular functions in strongly polynomial time, J. Combin. Theory Ser. B 80 (2000), 346-355.

[25] A. Shioura, Fast scaling algorithms for M-convex function minimization with application to the resource allocation problem, Tohoku University, (2002).

[26] A. Tamura, Coordinatewise domain scaling algorithm for M-convex function minimization, in: "Proceedings of the Ninth Conference on Integer Programming and Combinatorial Optimization," Lecture Notes in Computer Science Vol. 2337 (W. J. Cook and A. S. Schulz, Eds.) pp. 21-35, Springer-Verlag, Berlin, 2002. 